\title{
An Assessment of Ahara as a Nidana W.S.R to Charaka Samhita
}

\author{
Review article
}

$\begin{array}{r}\text { Hema Sundari Chinnam }{ }^{\mathbf{*}^{*}} \text {, Shivudu KV } \mathbf{K}^{\mathbf{2}} \text {, Ramreddy } \text { GP }^{\mathbf{3}} \\ \text { 1. P.G.Scholar, 2. Reader, 3. Prof \& H.O.D, } \\ \text { Department of Basic principles, S.V. Ayurvedic College, Tirupati. } 517507 \\ \hline\end{array}$

\begin{abstract}
Ayurveda, the art of living deals with all aspects of life from birth to death. Human life is supported by three pillars-Ahara, Nidra, Brahmacharya(1). The causes of the diseases are mentioned as Asatmyaendriyarthasamyoga, Pragnaparadha and Parinama(2) etc i.e. improper Ahara, Vihara (food habits and lifestyles). In this era of modernization, the lifestyle of people is changed significantly. Factors like high calorie food, stress, irregular dietary habits etc contribute to life style disorders. This is the time to explore the secrets, concepts and principles mentioned in Charaka Samhita. Aims and Objectives: 1. Critical evaluation of percentage of Aharaja factors in causing diseases and there by establishing the need for attention of Ahara. 2. Listing of Top 10 diseases caused by predominance of Ahara, Vihara, Manasika and other factors. 3. Catalogue of the complete Aharaja Nidana capable of producing different diseases in one umbrella. Materials and methods: The entire Nidanas mentioned in Charaka Samhita were divided into 4 groups-- Aharaja, Viharaja, Manasika and Others. Observations and results: 94 disease conditions were dealt along with specific Nidana and common Nidana completely from the entire Charaka Samhita. 269 Aharaja nidana were mentioned for 91 diseases as a whole.
\end{abstract}

Keywords: Nidana, Aharaja nidana, Viharaja nidana, Manasika nidana, Charaka samhita.

\section{Introduction:}

Ayurveda is reflective in its treasures and principles as antiquity goes back to Vedas which are the original sources of the subject matter of Ayurveda. The available literature is in the form of Samhita which means "Samyak Hitam Pratipadyam Yashyaha" (Vachaspatyam) and "compilation of knowledge". Only a few Samhitas are available today in a better state and the Charaka Samhita enjoys the place of moon among the stars. Charaka Samhita has exercised its

*Corresponding Author:

Hema Sundari. Ch,

P.G.Scholar-Final year,

S.V.Ayurvedic College,

SVIMS Road,Tirupati,Andhra Pradesh

Mobile no: +91-7396498853

Email:drhemasundari@gmail.com sovereignty by virtue of its time proven principles and approach of disease manifestation and treatment principles.

In this present era of modernization, people are neglecting the causative factors for the diseases and rushing towards treatment methodologies. It's the need of the hour to concentrate on the concept of "Nidana parivarjana chikitsa(3)" i.e. towards causative factors. So the present study was taken to evaluate the role of Aharaja, Viharaja, Manasika and other Nidanas individually in the manifestation of disease and particularly Ahara.

\section{Aims and objectives:}

1. Critical evaluation of percentage of Aharaja factors in causing diseases and there by establishing the need for attention of Ahara. 
2. Listing of Top 10 diseases caused by predominance of Ahara, Vihara, Manasika and other factors.

3. Catalogue of the complete Aharaja Nidana capable of producing different diseases in one umbrella.

\section{Materials and methods:}

The entire disease conditions in Charaka samhita having specific and common Nidana were taken and in each disease the factors are arranged as follows:

1. The factors allied to diet and dietetics are grouped in Aharaja Nidana.
2. The factors related to Vihara i.e Vega Dharana or the activities under Viharaja nidana.

3. The psychological or emotional factors or Manasika Bhavas are positioned in Manasika Nidana.

4. The rest of the Nidana are grouped in others.

The assessment of percentage of each group i.e.Aharaja nidana, Viharaja nidana, Manasika nidana and others factors involved in each disease is done by dividing the number of factors involved in each group by the total number of factors and then multiplied by hundred.

Observations:

\begin{tabular}{|c|c|c|c|c|}
\hline Disease & Aharaja & Viharaja & Manasika & Others \\
\hline 1.Vata Jwara & $24 \%$ & $35 \%$ & $12 \%$ & $29 \%$ \\
\hline 2.Pitta Jwara & $67 \%$ & $25 \%$ & $8 \%$ & $0 \%$ \\
\hline 3. Kapha Jwara & $70 \%$ & $20 \%$ & $10 \%$ & $0 \%$ \\
\hline $\begin{array}{l}\text { 4.Dwandwa Sannipata } \\
\text { Jwara }\end{array}$ & $27.8 \%$ & $66.7 \%$ & $0 \%$ & $5.5 \%$ \\
\hline 5.Raktapitta & $98.33 \%$ & $1.66 \%$ & $0 \%$ & $0 \%$ \\
\hline 6.Gulma & $18.18 \%$ & $27.27 \%$ & $9 \%$ & $46 \%$ \\
\hline 7. Vataja Gulma & $37 \%$ & $25 \%$ & $13 \%$ & $25 \%$ \\
\hline 8.Pittaja Gulma & $58 \%$ & $17 \%$ & $8 \%$ & $17 \%$ \\
\hline 9.Kaphaja Gulma & $67 \%$ & $33 \%$ & $0 \%$ & $0 \%$ \\
\hline 10.Prameha & $72.72 \%$ & $27.27 \%$ & $0 \%$ & $0 \%$ \\
\hline 11.Kaphaja Prameha & $86 \%$ & $14 \%$ & $0 \%$ & $0 \%$ \\
\hline 12.Pittaja Prameha & $64 \%$ & $27 \%$ & $9 \%$ & $0 \%$ \\
\hline 13.Vataja Prameha & $32 \%$ & $27 \%$ & $9 \%$ & $32 \%$ \\
\hline 14.Kusta & $63 \%$ & $20 \%$ & $0 \%$ & $17 \%$ \\
\hline 15.Switra & $14 \%$ & $0 \%$ & $57 \%$ & $29 \%$ \\
\hline 16. Rajayakshma & $25 \%$ & $50 \%$ & $0 \%$ & $25 \%$ \\
\hline 17.Unmada & $26 \%$ & $4 \%$ & $44 \%$ & $26 \%$ \\
\hline 18.Vataja Undmada & $57 \%$ & $0 \%$ & $14 \%$ & $29 \%$ \\
\hline 19.Pittaja Unmada & $100 \%$ & $0 \%$ & $0 \%$ & $0 \%$ \\
\hline 20.Kaphaja Unmada & $50 \%$ & $50 \%$ & $0 \%$ & $0 \%$ \\
\hline 21.Apasmara & $31 \%$ & $4 \%$ & $48 \%$ & $17 \%$ \\
\hline 22.Swayathu & $38 \%$ & $14 \%$ & $0 \%$ & $48 \%$ \\
\hline 23.Kshataksheena & $16 \%$ & $84 \%$ & $0 \%$ & $0 \%$ \\
\hline 24.Udara & $32 \%$ & $14 \%$ & $0 \%$ & $54 \%$ \\
\hline 25.Vataaja Udara & $34 \%$ & $33 \%$ & $0 \%$ & $34 \%$ \\
\hline 26.Pittaja Udara & $80 \%$ & $20 \%$ & $0 \%$ & $0 \%$ \\
\hline 27.Kaphaja Udara & $78 \%$ & $22 \%$ & $0 \%$ & $0 \%$ \\
\hline 28.Sannipata Udara & $30 \%$ & $0 \%$ & $0 \%$ & $70 \%$ \\
\hline 29.Pleehodara & $0 \%$ & $67 \%$ & $0 \%$ & $33 \%$ \\
\hline
\end{tabular}




\begin{tabular}{|c|c|c|c|c|}
\hline 30.Baddhagudodara & $20 \%$ & $0 \%$ & $0 \%$ & $80 \%$ \\
\hline 31.Chidrodara & $83 \%$ & $17 \%$ & $0 \%$ & $0 \%$ \\
\hline 32.Jalodara & $100 \%$ & $0 \%$ & $0 \%$ & $0 \%$ \\
\hline 33.Arsas & $73 \%$ & $16 \%$ & $0 \%$ & $11 \%$ \\
\hline 34.Vataja Arsas & $56 \%$ & $25 \%$ & $6 \%$ & $13 \%$ \\
\hline 35.Pittaja Arsas & $54 \%$ & $20 \%$ & $13 \%$ & $13 \%$ \\
\hline 36. Kaphaja Arsas & $43 \%$ & $36 \%$ & $7 \%$ & $14 \%$ \\
\hline 37.Agnidusti & $53 \%$ & $6 \%$ & $0 \%$ & $41 \%$ \\
\hline 38. Vataja Grahani & $70 \%$ & $30 \%$ & $0 \%$ & $0 \%$ \\
\hline 39.Pittaja Grahani & $100 \%$ & $0 \%$ & $0 \%$ & $0 \%$ \\
\hline 40.Kaphaja Grahani & $80 \%$ & $20 \%$ & $0 \%$ & $0 \%$ \\
\hline 41.Pandu & $48 \%$ & $19 \%$ & $24 \%$ & $9 \%$ \\
\hline 42. Hikkaswasa & $44 \%$ & $15 \%$ & $0 \%$ & $41 \%$ \\
\hline 43.Vataja Kasa & $62 \%$ & $38 \%$ & $0 \%$ & $0 \%$ \\
\hline 44.Pittaja Kasa & $62 \%$ & $25 \%$ & $13 \%$ & $0 \%$ \\
\hline 45.Kaphaja Kasa & $67 \%$ & $33 \%$ & $0 \%$ & $0 \%$ \\
\hline 46.Kshtaja Kasa & $0 \%$ & $100 \%$ & $0 \%$ & $0 \%$ \\
\hline 47.Kshayajakasa & $40 \%$ & $40 \%$ & $20 \%$ & $0 \%$ \\
\hline 48. Vataja Atisara & $44 \%$ & $56 \%$ & $0 \%$ & $0 \%$ \\
\hline 49.Pittaja Atisara & $55 \%$ & $27 \%$ & $18 \%$ & $0 \%$ \\
\hline 50.Kaphaja Atisara & $67 \%$ & $16 \%$ & $17 \%$ & $0 \%$ \\
\hline 51.Sannipataja Atisara & $41 \%$ & $24 \%$ & $9 \%$ & $26 \%$ \\
\hline 52.Vataja Chardi & $33 \%$ & $17 \%$ & $33 \%$ & $17 \%$ \\
\hline 53.Pittaja Chardi & $100 \%$ & $0 \%$ & $0 \%$ & $0 \%$ \\
\hline 54.Kaphaja Chardi & $60 \%$ & $20 \%$ & $0 \%$ & $20 \%$ \\
\hline 55.Sannipataja Chardi & $33 \%$ & $0 \%$ & $0 \%$ & $67 \%$ \\
\hline 56.Agantuja Chardi & $57 \%$ & $29 \%$ & $14 \%$ & $0 \%$ \\
\hline 57. Visarpa & $75 \%$ & $5 \%$ & $0 \%$ & $20 \%$ \\
\hline 58.Vataja Visarpa & $67 \%$ & $0 \%$ & $0 \%$ & $33 \%$ \\
\hline 59.Pittaja Visarpa & $100 \%$ & $0 \%$ & $0 \%$ & $0 \%$ \\
\hline 60.Kaphaja Visarpa & $83 \%$ & $17 \%$ & $0 \%$ & $0 \%$ \\
\hline 61.Grandhi Visarpa & $78 \%$ & $0 \%$ & $11 \%$ & $11 \%$ \\
\hline 62.Trishna & $47 \%$ & $11 \%$ & $26 \%$ & $16 \%$ \\
\hline 63. Udavarta & $71 \%$ & $29 \%$ & $0 \%$ & $0 \%$ \\
\hline 64.Mutrakricchra & $67 \%$ & $33 \%$ & $0 \%$ & $0 \%$ \\
\hline 65.Hridroga & $8 \%$ & $15 \%$ & $23 \%$ & $54 \%$ \\
\hline 66. Vataja Hridroga & $67 \%$ & $16 \%$ & $17 \%$ & $0 \%$ \\
\hline 67.Pittaja Hridroga & $78 \%$ & $11 \%$ & $11 \%$ & $0 \%$ \\
\hline 68. Kaphaja Hridroga & $50 \%$ & $33 \%$ & $17 \%$ & $0 \%$ \\
\hline 69.Pratisyaya & $7 \%$ & $72 \%$ & $7 \%$ & $14 \%$ \\
\hline 70.Arochaka & $15 \%$ & $14 \%$ & $57 \%$ & $14 \%$ \\
\hline 71.Urustambha & $75 \%$ & $15 \%$ & $5 \%$ & $5 \%$ \\
\hline 72.Vatavyadhi & $20 \%$ & $47 \%$ & $13 \%$ & $20 \%$ \\
\hline 73. Vatarakta & $73 \%$ & $19 \%$ & $2 \%$ & $6 \%$ \\
\hline 74. Yonivyapath & $25 \%$ & $25 \%$ & $0 \%$ & $60 \%$ \\
\hline 75.Sukradusti & $26 \%$ & $30 \%$ & $18 \%$ & $26 \%$ \\
\hline 76.Beejopaghataja & $33 \%$ & $14 \%$ & $24 \%$ & $29 \%$ \\
\hline
\end{tabular}




\begin{tabular}{|l|l|l|l|l|}
\hline Klaibya & & & & \\
\hline $\begin{array}{l}\text { 77.Dhwajabhangaja } \\
\text { Klaibya }\end{array}$ & $39 \%$ & $39 \%$ & $0 \%$ & $22 \%$ \\
\hline $\begin{array}{l}\text { 78.Jarasambhavaja } \\
\text { Klaibya }\end{array}$ & $33.3 \%$ & $33.3 \%$ & $0 \%$ & $33.3 \%$ \\
\hline 79.Sukrakshayaja Klaibya & $38 \%$ & $0 \%$ & $62 \%$ & $0 \%$ \\
\hline 80.Raktapradara & $100 \%$ & $0 \%$ & $0 \%$ & $0 \%$ \\
\hline 81.Stanyadusti & $63 \%$ & $17 \%$ & $10 \%$ & $10 \%$ \\
\hline 82.Siroroga & $16 \%$ & $56 \%$ & $4 \%$ & $24 \%$ \\
\hline 83. Vataja Siroroga & $11 \%$ & $50 \%$ & $17 \%$ & $22 \%$ \\
\hline 84.Pittaja Siroroga & $57 \%$ & $29 \%$ & $14 \%$ & $0 \%$ \\
\hline 85. Kaphaja Siroroga & $60 \%$ & $40 \%$ & $0 \%$ & $0 \%$ \\
\hline 86. Krimija Siroroga & $75 \%$ & $0 \%$ & $0 \%$ & $25 \%$ \\
\hline 87. Ardhavabhedaka & $33 \%$ & $67 \%$ & $0 \%$ & $0 \%$ \\
\hline 88.Anantavata & $80 \%$ & $20 \%$ & $0 \%$ & $0 \%$ \\
\hline 89.Suryavarta & $0 \%$ & $50 \%$ & $0 \%$ & $0 \%$ \\
\hline 90.Tandra & $43 \%$ & $14 \%$ & $29 \%$ & $14 \%$ \\
\hline 91.Sthoulya & $36 \%$ & $46 \%$ & $9 \%$ & $9 \%$ \\
\hline 92. Karshya & $29 \%$ & $36 \%$ & $14 \%$ & $21 \%$ \\
\hline 93.Antarvidradhi & $68 \%$ & $32 \%$ & $0 \%$ & $0 \%$ \\
\hline 94.Raktadusti & $69 \%$ & $19 \%$ & $2 \%$ & $10 \%$ \\
\hline
\end{tabular}

\section{Top 10 Aharaja nidana predominant diseases:}

Raktapradara (100\%), Pittaja Visarpa (100\%), Pittaja Chardi (100\%), Pittaja Grahani (100\%), Jalodara (100\%), Pittaja Unmada (100\%), Raktapitta (98.3\%), Kaphajaprameha (86\%), Chidrodara (83\%), Kaphajavisarpa (83\%), Kaphajagrahani (80\%), Pittajaudara (80\%), Kaphajaudara (78\%), Pittajahridroga (78\%), Grandhivisarpa (78\%), Krimijasiroroga (75\%), Urustambha (75\%), Visarpa (75\%), Vatarakta (73\%), Arsas (73\%), Prameha (72.72\%), Udavarta (70\%).

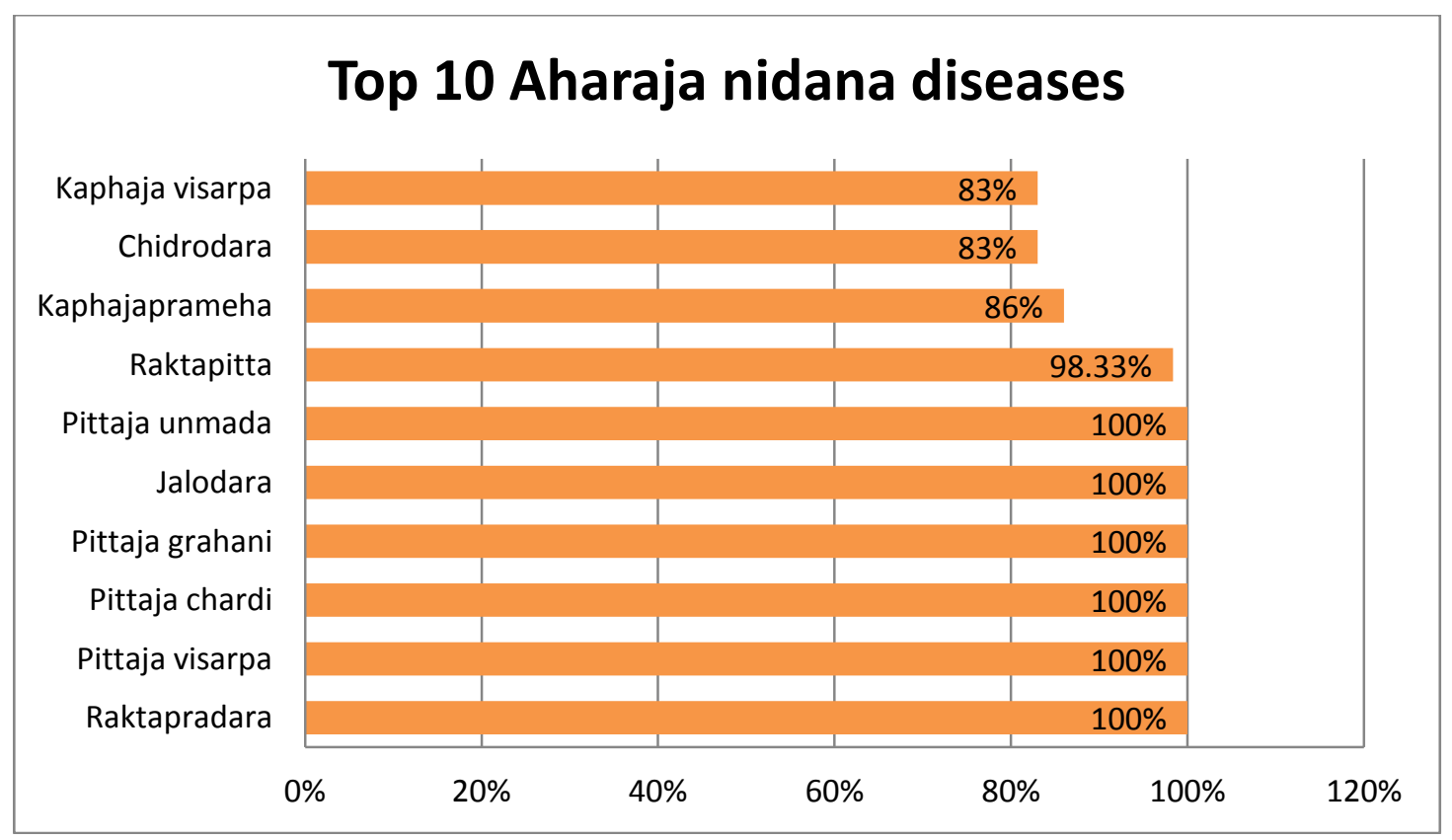




\section{Top 10 Viharaja Nidana predominant diseases:}

Kshatajakasa (100\%), Kshataksheena (84\%)Pratisyaya (72\%), Ardhavabhedaka (67\%), Pleehodara (67\%), Dwandwa Sannipataja Jwara, Siroroga (56\%), Vataja Atisara (56\%) Suryavarta (50\%), Vataja Siroroga (50\%), Kaphaja Unmada (50\%), Rajayakshma (50\%) Vatavyadhi (47\%), Sthoulya (46\%), Kaphaja Siroroga (40\%), Kshayajakasa (40\%).

\section{Top 10 Viharaja nidana diseases}

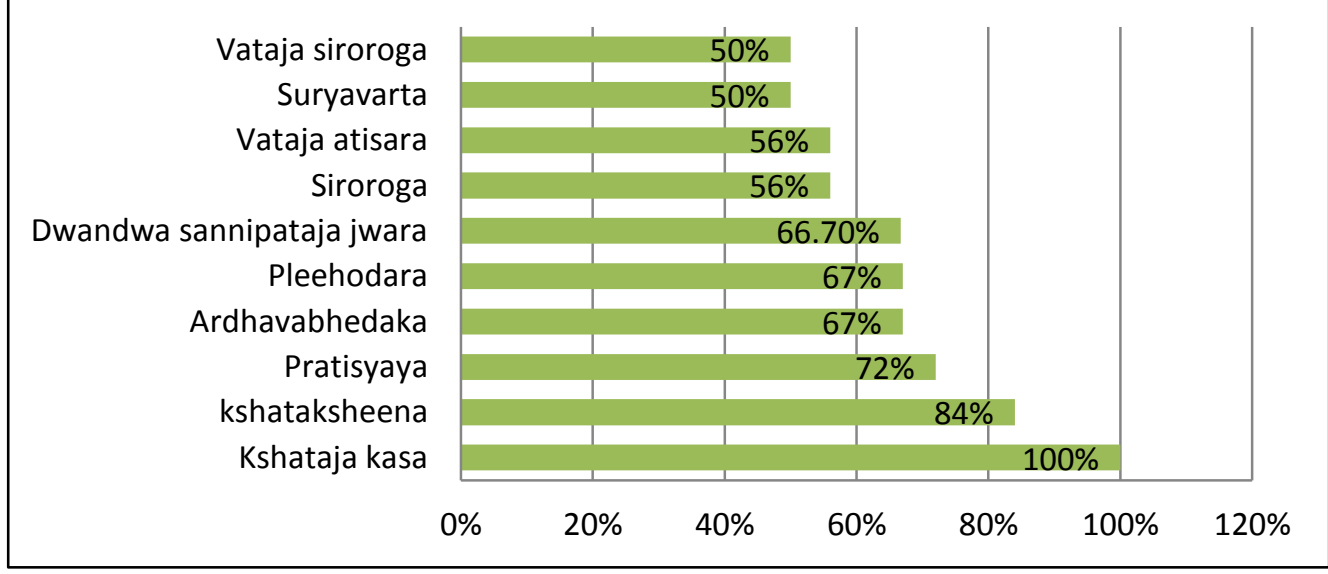

\section{Top 10 Manasika Nidana predominant diseases:}

Sukrakshayaja Klaibya(62\%), Arochaka(57\%), Switra ((57\%), Apasmara(48\%), Unmada(44\%), Vataja Chardi(33\%), Tandra(29\%), Trishna(26\%), Beejopaghataja Klaibya(24\%), Pandu(24\%), Hridroga(23\%), Kshayaja Kasa(20\%).

It is known fact that psychological status has influence on impotency which was supported by the above table in Sukrakshayaja klaibya, Beejopaghataja Klaibya and heart diseases (Hridroga)

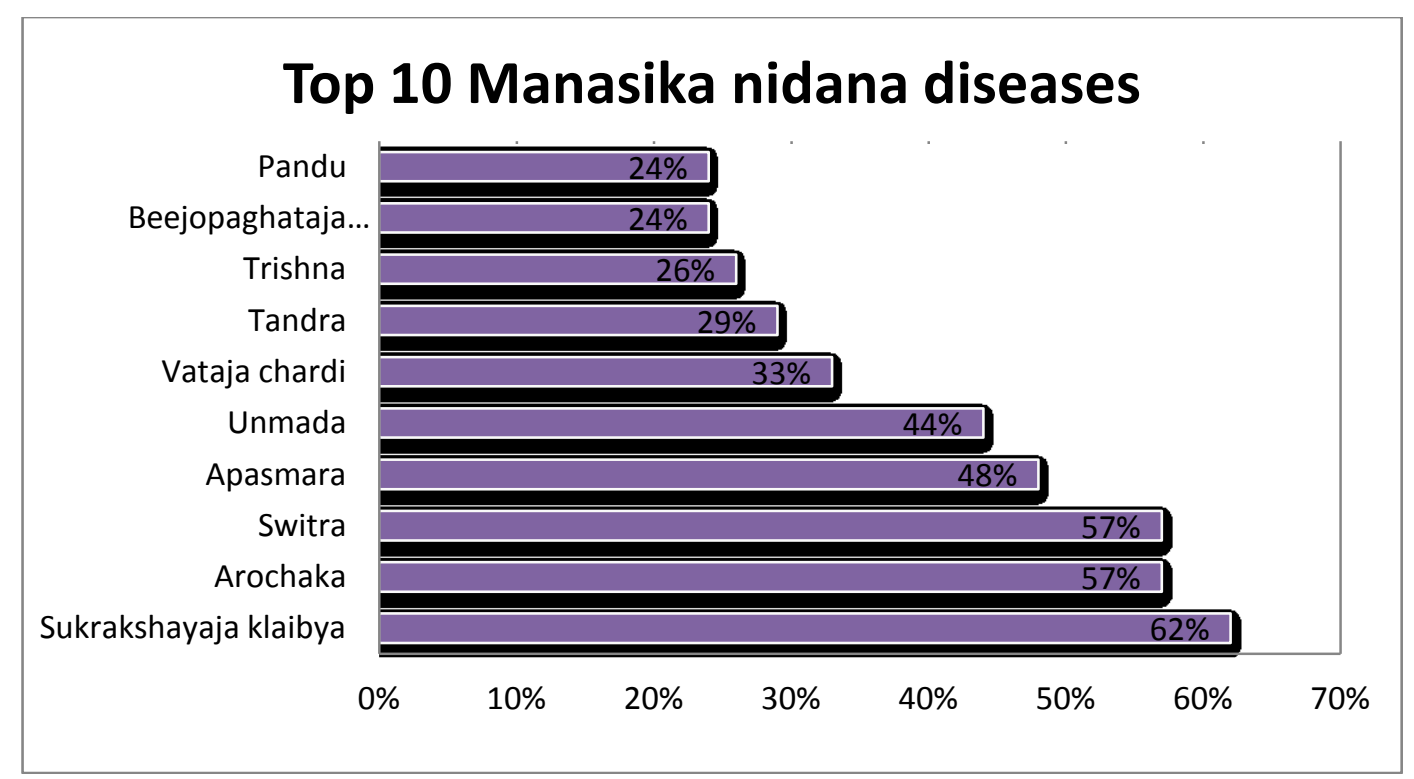




\section{Top 10 Other Nidana predominant diseases:}

Baddhagudodara(80\%), Sannipataja Udara(70\%), Sannipataja Chardi(67\%), Yonivyapath(60\%), Hridroga(54\%), Udara(54\%), Swayathu(48\%), Gulma(46\%), Hikkaswasa(41\%), Agnidusti(41\%), Vataja Udara(34\%), Jarasambhava Klaibya(33.3\%)

\section{TOP 10 OTHER NIDANA DISEASES}

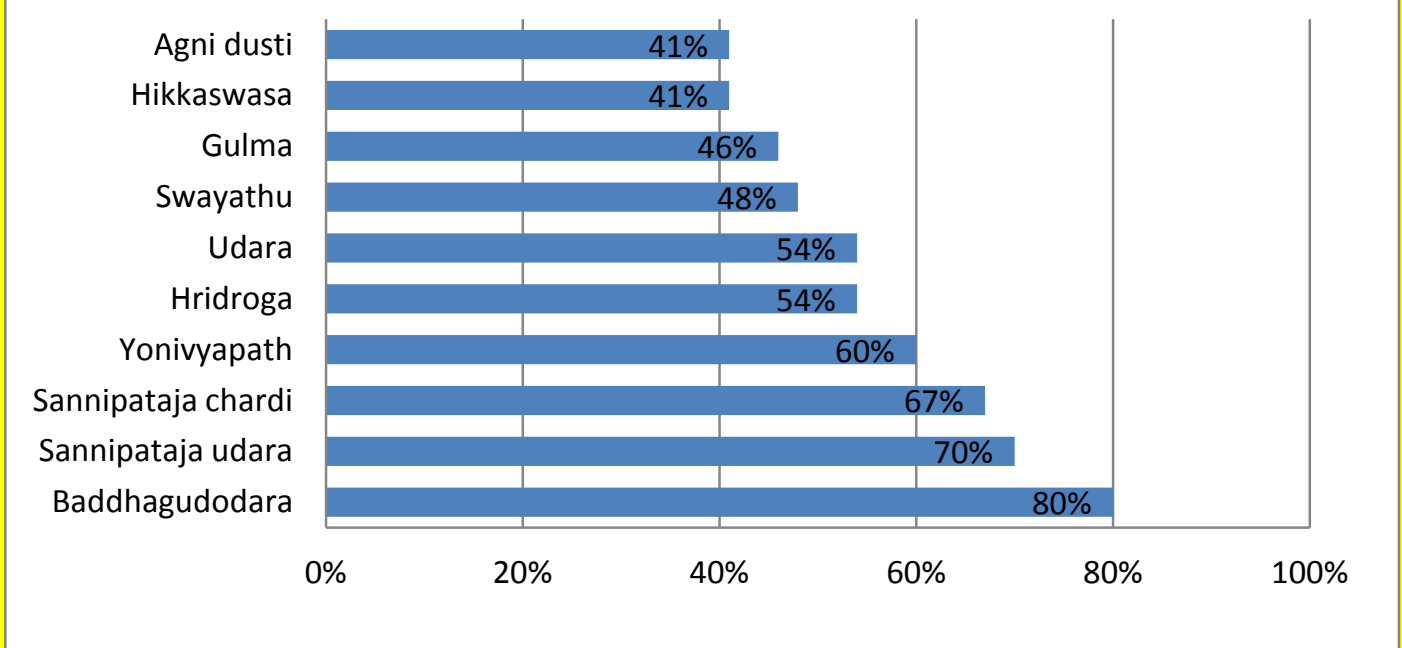

\section{Assessment of four nidanas in entire diseases:}

- Aharaja Nidana: It is involved in the manifestation of $91(97 \%)$ disease conditions and not involved in-3(3\%) diseases i.e Suryavarta, Kshtaja Kasa, Pleehodara

\section{Aharaja nidana in entire diseases}

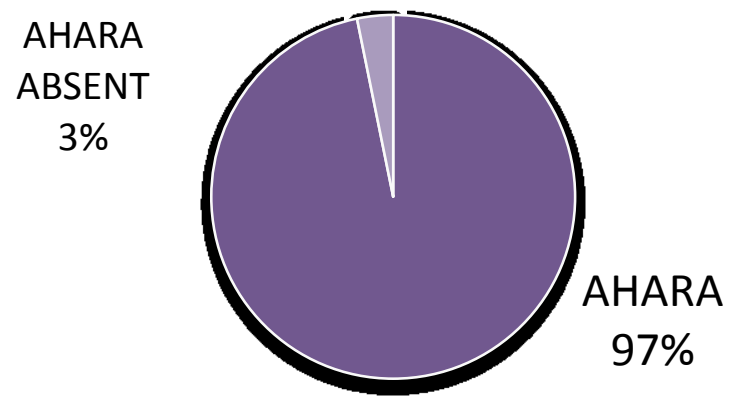

- Viharaja Nidana: Viharaja nidana is involved in $79(84 \%)$ disease conditions and not involved in 15(16\%) diseases. They are Krimija Siroroga, Raktapradara, Sukrakshayaja Klaibya, Grandhi Visarpa, Pittaja Visarpa, Vataja Visarpa, 
Sannipataja Chardi, Pittaja Chardi, Pittaja Grahani, Jalodara, Baddhagudodara, Sannipata Udara, Pittaja Unmada, Vataja Unmada, Switra.

\section{Viharaja nidana in entire diseases}

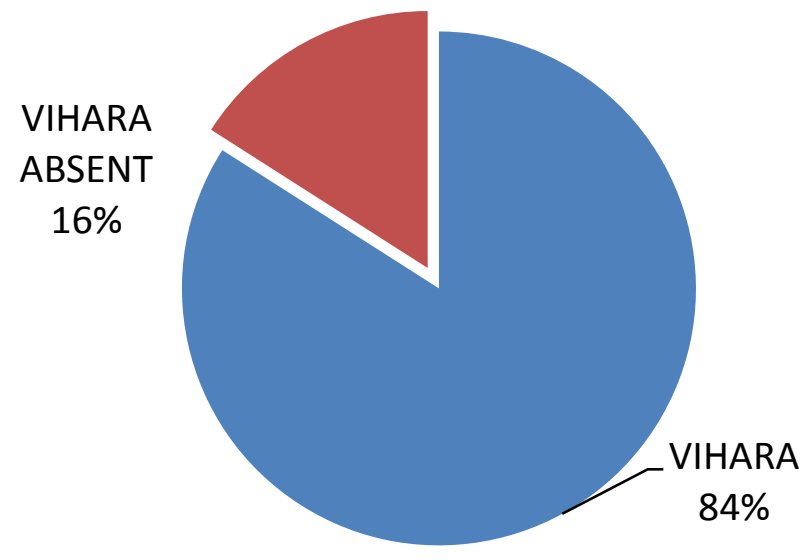

- Manasika nidana:Manasika nidana is present in 77 (88\%) disease conditions: In 17(18\%) diseases Manasika Nidana is not involved. Antarvidradhi, Kaphaja Gulma, Suryavarta, Anantavata, Ardhavabhedaka, Krimija Siroroga, Kaphaja Siroroga, Raktapradara, Jarasambhava Klaibya, Dhwajabhanga Klaibya, Yonivyapath, Mutrakricchra, Udavarta, Kaphaja Visarpa, Pittaja Visarpa, Kaphaja Chardi.

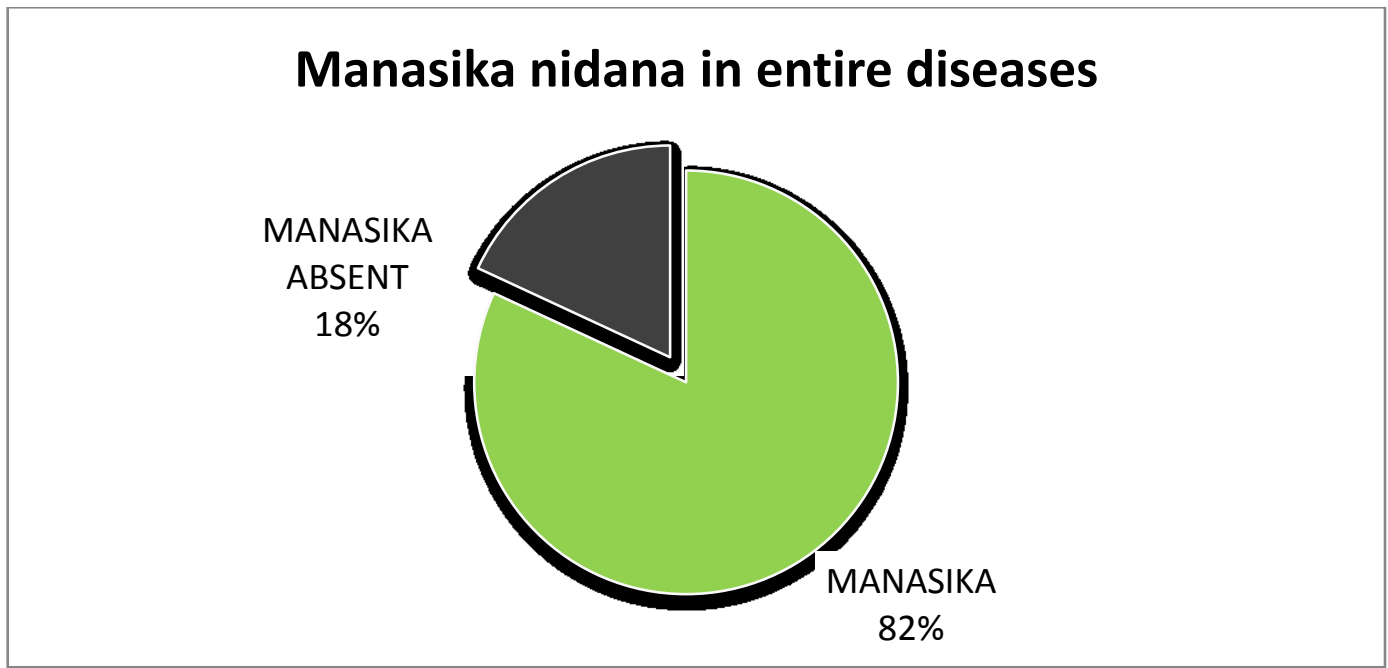

- Other nidana: Other nidana is present in 52 disease conditions and not involved in 42(44.6\%) disease conditions.They are Antarvidradhi, Kaphaja Gulma, Suryavarta, Anantavata, Ardhavabhedaka, Kaphaja Siroroga, Pittaja Siroroga, Raktapradara, Sukrakshayaja Klaibya, Kaphaja Hridroga, Pittaja Hridroga, Vataja Hridroga, Mutrakricchra, Udavarta, Kaphaja Visarpa, Pittaja Visarpa, Agantuja Chardi, Pittaja Chardi, Raktapitta, Kaphaja Atisara, Pittaja Atisara, Vataja Atisara, Kshayajakasa, Kshataja Kasa, Kaphaja Kasa, PittajaKasa, Vataja Kasa, Kaphaja 
Grahani, Pittaja Grahani, Vataja Grahani, Jalodara, Chidrodara, Kapha Jwara, Kaphaja Udara, Pittaja Udara, Kshataksheena, Kaphaja Unmada, Pitta Jwara, Pittaja Unmada, Pittaja Prameha, Kaphaja Prameha, Prameha

\section{Other nidana in entire diseases}

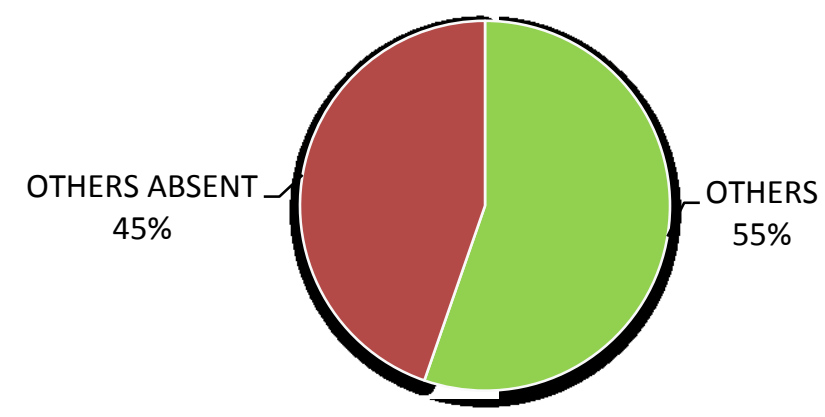

The following diet is able to cause many diseases:

1. Abhisyandi:Doshaja arsa, Stanyadosha, Hikkaswasa, Kaphaja kasa, Grandhi visarpa--5

2. Adhyasana:Kusta, Visarpa, Mutrakrichchra, Urustambha, Ardhavabhedaka, Pittaja udara--6

3. Ahita Bhojana:Apasmara--1

4. Aja mamsa: Doshaja arsa--1

5. Ajeernasana:Pittja Jwara, Pittaja Prameha, Kusta, Doshaja Arsa, Agnidusti, Visarpa, Mutrakrichcchra, Pratisyaya, Vatarakta, Beejopaghataja Klaibya, Stanya Dosha, Krimija Siroroga, Antarvidradhi, Pittaja unmada, Pittajaudara, Pittaja grahani, Pittaja chardi, Pittaja hridroga--18

6. Alpa Bhojana: Ananta Vata, Beejopaghataja Klaibya, Vatarakta, Vatavyadhi, Vataja unmada, Kshataksheena, Vataja kasa, Vataja atisara, Vataja hridroga--9

7. Amaksheera:Hikkaswasa---1

8. Amamulaka:Doshaja Arsa--1

9. Amapadartha sevana:Nijaswayathu-1

10. Amedhya:Agantuja chardi--1
11. Amla mastu:Visarpa, Raktadusti, Raktapradara--3

12. Amlakanji- processed with Yavaka, Uddalaka, Kora Dusha, Nishpava, Masha, Kuluttha-Raktapitta--1

13. Amlakanji: Raktapitta--1

14. Amlarasa:Pittaja Jwara, Kaphaja Jwara, Pittaja Gulma, Raktapitta, Kusta, Udara, Nijaswayathu, Pandu, Visarpa, Trishna, Vatarakta, Sukradusti, Dhwajabhangaja Klaibya, Raktapradara, Stanyadosha, Siroroga, Raktadusti, Pittaja unmada, Vataja udara, Pittaja udara, Kaphaja arsa, Pittaja Grahani, Pittaja kasa, Pittaja atisara, Pittaja chardi, Pittaja visarpa, Kaphaja Visarpa, Pittaja hridroga---28

15. Anasana:Vataja Jwara, Vataja Gulma, Vataja Prameha, Dwandwa Sannipataja Jwara, Agnidusti, Sannipataja Atisara, Udavarta, Vatavyadhi, Vata Rakta, Beejopaghataja Klaibya, Vataja grahani--11

16. Annaparivarta:

Dwandwasannipataja jwara--1

17. Anuchita Ahara:Unmada--1 
18. Anupa mamsa:Prameha, Kaphaja prameha, Nija swayathu, Hikkaswasa, Visarpa, Mutrakricchra, Urustambha, Vatarakta, Dhwajabhangaja klaibya, Stanya dosha, Raktadusti, Kaphaja udara--12

19. Aranala:Vatarakta --1

20. Asatmya bhojana: Doshaja Arsa, Agnidusti, Pandu, Kshayaja Kasa, Sannipataja Atisara, Visarpa, Sukradusti, Dhwajabhangaja Klaibya, Sukrakshayaja Klaibya, Stanya Dosha, Antarvidradhi--11

21. Asuchi Ahara: Udara, Unmada, Apasmara--3

22. Asuchi anna:Unmada, Apasmara, Udara, Agantuja chardi-4

23. Ati khara: Sannipataja Atisara--1

24. Atibhojana: Agnidusti, Antarvidradhi, Kaphaja grahani, Kaphaja hridroga--4

25. Atikatina:Sannipataja Atisara--1

26. Atikrisa-atyambupana:Jalodara--1

27. Atimadyapana:Sannipataja atisara, Antarvidradhi, Stanyadosha, Trishna, Visarpa, Pittaja gulma, Pittaja arsa, Pittaja hridroga -- 8

28. Atiseetambu sevana:Siroroga, Hikkaswasa--2

29. Atyadana:Visarpa, Ardhavabhedaka, Stanya Dosha -3

30. Avika mamsa: Doshaja arsa--1

31. Avikamamsa- processed with Yavaka, Uddalaka, Kora Dusha, Nishpava, Masha Kuluttha Raktapitta --1

32. Badaramla: Raktapitta--1

33. Bahu ushna madya: Raktadusti--1

34. Bahuteekshna madya: Raktadusti--1

35. Bhustrna: Raktapitta--1

36. Bisa: Doshaja arsa--1

37. Cheenaka: Kaphaja Prameha--1

38. Chilichima with milk:Kusta --1

39. Dadhi Manda:Raktapitta, Doshaja Arsa--2

40. Dadhi with Kola/kuluttha/masha/atasi/kusumbha sneha:Kusta--1
41. Dadhi:Raktapitta,

Prameha, Kapahaja Prameha, Kusta, Nijaswayathu, Hikkaswasa, Visarpa, Urustambha, Vatarakta, Dhwajabhanagaja Klaibya, Raktapradara, Stanya Dosha, Raktadusti, Kaphaja udara, Drava:Kaphaja prameha, Kusta, Urustambha--14

42. Durbala prakriti nirahara: Sukrakshayaja Klaibya--1

43. Durbalagni amapadartha sevana:Udara--1

44. Durbalagni apathyahara sevana:Udara--1

45. Durbalagni Viruddha padartha sevana:Udara--1

46. Durbalaprakriti nirahara:

Sukrakshayaja Klaibya--1

47. Dustanna:Unmada, Agnidusti, Nija Swayathu--3

48. Gandeera: Raktapitta--1

49. Gara: Dwandwasannipataja jwara, Udara, Nija swayathu--3

50. Gavyamamsa- processed with Yavaka, Uddalaka, Kora Dusha, Nishpava, Masha, KulutthaRaktapitta, Kusta--1

51. Gavyamamsa:Doshaja arsa--1

52. Gramya mamsa:Prameha, Kaphaja prameha, Visarpa, Urustambha, Raktapradara, Stanyadosha--6

53. Guda:Prameha, Kusta, Dhwajabhangaja Klaibya, Sthanyadosha, Krimija Siroroga ---5

54. Guru saka: Doshaja arsa--1

55. Guru:Kusta, Nijaswayathu, Arsa, Agnidusti, Hikkaswasa, Sannipataja Atisara, Raktapradara, Siroroga, Sthoulya, Tandra, Kaphaja Jwara, Kaphaja Gulma, Kaphaja arsa, Kaphaja grahani, Kaphaja kasa, Kaphaja atisara, Kaphaja Chardi, Grandhi visarpa, Kaphaja visarpa, Kaphaja hridroga --20

56. Gurusaka:Doshaja arsa--1

57. Gurusalilapana:Doshajaarsa--1

58. Harita:Raktadusti, Siroroga--2

59. Haritamardaka: Doshaja arsa--1 
60. Haayanaka: Kaphaja Prameha--1

61. Ikshu rasa:Doshaja arsa, Vatarakta$-2$

62. Ikshu vikara:Kaphajaprameha--1

63. Jalajamamsa:Hikkaswasa, Raktadusti, Kapahaja udara --3

64. Jambava with unboiled milk: Raktapitta --1

65. Jambava: Raktapitta --1

66. Kalamalaka: Raktapitta--1

67. Kalateetabhojana:Sannipataja Atisara --1

68. Karanja:Raktapitta--1

69. Kaseruka: Doshaja arsa--1

70. Kashayarasa: Vataja prameha, Vatarakta, Udavarta, Sukradusti, Vataja arsa, Vataja grahani, Vataja kasa--7

71. Katina:Grandhi visarpa--1

72. Katurasa:Pittaja Jwara, Pittaja Gulma, Pittaja Prameha, Vataja Prameha, Raktapitta, Visarpa, Trishna, Udavarta, Vatarakta, Raktapradara, Stanya Dosha, Rakatdusti, Pittaja unmada, Pittaja udara, Vataja arsa, Pittaja arsa, Vataja grahani, Pittaja grahani, Pittaja kasa, Pittaja kasa, Pittaja atisara, Pittaja chardi, Pittaja hridroga--23

73. Katwara- processed with Yavaka, Uddalaka, Kora Dusha, Nishpava, Masha, Kuluttha-Raktapitta--1

74. Khadayusha: Raktapitta--1

75. Kilata:Doshaja arsa--1

76. Koradusha:Raktapitta, Kusta--2

77. Krisa mamsa: Doshaja arsa--1

78. Krisara:Kaphajaprameha, Raktapradara, Stanyadosha--3

79. Krounchadana: Doshaja arsa--1

80. Krusova ruksha anna sevana: Sukrakshayaja Klaibya--1

81. Krusova ruksha aushadha sevana: Sukrakshayaja Klaibya--1

82. Krusova ruksha pana sevana: Sukrakshayaja Klaibya--1

83. Kshara:Udara, Pandu, Raktadusti, Stanyadosha, Pittaja Jwara, Pittaja Prameha, Vatarakta, Trishna,
Dhwajabhanga Klaibya, Pittaja arsa, Pitaja grahani, Pittaja kasa, Pittaja atisara, Pittaja hridroga--14

84. Kshavaka: Raktapitta--1

85. Ksheena-atyambupana:Jalodara--1

86. Ksheeramodaka:Doshajaarsa--1

87. Kuluttha:Raktapitta, Kusta, Visarpa, Vatarakta, Raktadusti--5

88. Kurchika:Visarpa--1

89. Kuteraka: Raktapitta--1

90. Kuvara: Raktapitta--1

91. Laghu Ahara while jeernajeerne samsnata:Urustambha--1

92. Laghu anna: Vatavyadhi, Vataja Jwara, Vataja Prameha, Vataja arsa--4

93. Lakucha while indigestion: Raktapitta--1

94. Lakucha: Raktapitta--1

95. Langhana:Urustambha, Vatavyadhi, Karshya, Trishna, Vataja arsa-5

96. Lasuna:Raktapitta, Doshaja Arsa, Visarpa--3

97. Lavana rasa: Pittaja Jwara, Kaphaja Jwara, Pittaja Prameha, Raktapitta, Kusta, Nija Swayathu, Udara, Visarpa, Trishna, Vatarakta, Sukradusti, Dhwajabhangaja Klaibya, Raktapradra, Stanya Dosha, Raktadusti, Pittaja udara, Pittaja arsa, Kaphaja arsa, Pittaja atisara, Kaphaja visarpa, Pittaja hridroga--21

98. Losta bhakshana:Nijaswayathu--1

99. Madhu:Kusta--1

100. Madhulaka: Raktapitta--1

101. Madhurarasa:Kaphajaprameha, Doshajaarsa, Sthoulya, Tandra, Kaphaja jwara, Kaphaja udara, Kaphaja arsa, Kaphaja kasa, Kaphaja atisara, Grandhi visarpa,

102. Kaphaja visarpa--11

103. Madhusigru: Raktapitta--1

104. Mahavreehi: Kaphaja Prameha--1

105. Mahisha mamsa- processed with Yavaka, Uddalaka, Kora Dusha, Nishpava, Masha, KulutthaRaktapitta --1

106. Mahisha mamsa:Doshaja Arsa--1 
107. Maireya: Raktapitta--1

108. Mandagni-atyambupana:Jalodara--1

109. Mandakatikranta adyapana:Doshaja arsa--1

110. Mandhaka dadhi:Visarpa, Stanya Dosha---2

111. Manoghnasana:Arochaka--1

112. Masha:Raktapitta, Kusta, Pandu, Hikkaswasa, Visarpa, Vatarakta, Rakta Dusti--7

113. Mashayusha:Doshaja Arsa--1

114. Matsya mamsa- processed with Yavaka, Uddalaka, Kora Dusha, Nishpava, Masha, KulutthaRaktapitta--1

115. Matsya while indigestion-Raktapitta$-1$

116. Matsya:Raktapitta, Kusta, Arsa, Mutrakricchra, Doshaja Arsa, Kusta, Mutrakricchra --7

117. Medaka: Raktapitta--1

118. Medhya:Raktapradara--1

119. Mistanna bhojana:Vatarakta --1

120. Mrinala: Doshaja arsa --1

121. Mrit +saka-Nijaswayathu --1

122. Mritpankabhakshana:Nijaswayathu-1

123. Mukundaka: Kaphaja Prameha --1

124. Mulaka while indigestion:Kusta--1

125. Mulaka:Raktapitta, Kusta, Vatarakta, Raktadusti--4

126. Naishadha: Kaphaja Prameha--1

127. Nava harenu: Kaphaja Prameha--1

128. Nava-Hayanaka, Yavaka, Cheenaka, Uddalaka, Naishedha, Itkata, Mukundaka, Mahavreehi, Pramodaka, Sugandhaka : Kaphaja Prameha--10

129. Nava mandaka: Kaphaja Prameha -1

130. Nava Uddalaka:Kaphajaprameha. -1

131. Nava Yavaka Ativelam:Kapahaja Prameha --1

132. Navaanna:Prameha, Kusta --2

133. Navamadya: Kaphaja Prameha --1

134. Navamasha Supa With Sarpi:Kapahaja Prameha --1

135. Navapana:Prameha --1
136. Navasimbhi:Doshaja arsa --1

137. Navasuka:Doshajaarsa--1

138. Nishpava:Raktapitta, Pandu, Hikkaswasa, Vatarakta, Raktadusti-5

139. Padamamsa: Doshaja arsa --1

140. Paistika:Doshaja arsa --1

141. Pakshmavale saha anna bhojana:Baddhagudodara--1

142. Palala:Kaphajaprameha, Vatarakta $--2$

143. Pancha karma karshita amla sevana: Nijaswayathu --1

144. Pancha karma karshita lavana sevana: Nijaswayathu --1

145. Pancha karmakarshita anupa mamsa sevana:Nijaswayathu--1

146. Pancha karmakarshita atiguru sevana:Nijaswayathu --1

147. Pancha karmakarshita haritaka sevana:Nijaswayathu --1

148. Pancha karmakarshita madya sevana:Nijaswayathu --1

149. Pancha karmakarshita mandaka sevana:Nijaswayathu--1

150. Pancha karmakarshita oudaka mamsa sevana:Nijaswayathu --1

151. Pancha karmakarshita phala sevana:Nijaswayathu --1

152. Pancha karmakarshita pistanna sevana:Nijaswayathu --1

153. Pancha karmakarshita raga sevana:Nijaswayathu --1

154. Pancha karmakarshitavirudha nava sami dhanya sevana : Nijaswayathu $-1$

155. Pancha karmakarshitavirudha nava sukadhanya sevana:Nijaswayathu--1

156. Pancha karmakarsita dadhi: Nijaswayathu --1

157. Pancha karmakarsita saka: Nijaswayathu --1

158. Paramanna:Stanyadosha, Doshaja arsa-2

159. Pariklinna anna:Stanya dosha, Visarpa --2

160. Parnasa: Raktapitta --1

161. Paryushita mamsa: Doshaja arsa--1 
162. Paya

with

Kola/Kuluttha/Masha/Atasi/Kusumb ha-Kusta --1

163. Paya:Prameha, Kaphaja Prameha, Krimija siroroga, Kaphaja udara --4

164. Payasa:Kaphajaprameha, Raktapradara --2

165. Phanijjaka: Raktapitta --1

166. Phanita:Kusta --1

167. Picchila:Kaphaja jwara, Kaphaja udara --2

168. Pindaluka- processed with Yavaka, Uddalaka, Kora Dusha, Nishpava, Masha, Kuluttha-Raktapitta --1

169. Pindaluka:Doshaja Arsa --1

170. Pinyaka- processed with Yavaka, Uddalaka, Kora Dusha, Nishpava, Masha, Kuluttha-Raktapitta--1

171. Pinyaka:Doshaja Arsa, Pandu, Hikkaswasa, Vatarakta--4

172. Pinyaka with unboiled milkRaktapitta--1

173. Pistanna: Raktapitta, Kaphaja Prameha, Kusta, Dhwajabhangaja Klaibya --4

174. Pradusta madya:Sannipataja atisara, Raktadusti --2

175. Pramitasana: Karshya, Doshaja Arsa, Kshataksheena, Vataja arsa, Vataja Grahani, Vataja kasa, Vataja atisara --7

176. Pramodaka: Kaphaja Prameha --1

177. Prasaha mamsa:Raktadusti --1

178. Pratipasana:Agantuja chardi --1

179. Puteenam bhakshana:Doshaja arsa, Raktadusti, Krimija siroroga, Agantuja chardi --4

180. Puti mamsa: Doshaja arsa --1

181. Raga: Doshaja arsa --1

182. Ruksha :Vataja Jwara, Vataja Gulma, Pittaja Gulma, Vataja Prameha, Gulma, Udara, Trishna, Agni Dusti, Hikka Swasa, Sannipataja Atisara, Udavarta, Vatavyadhi, Vatarakta, Sukra Dusti, Beejopaghataja Antarvidardhi, Ardhavabhedaka, Klaibya, Vatajauneda, Karshya, Vatajaunmada,
Anantavata,

Kshataksheena,
Vataja udara, Vataja arsa, Vatajagrahani Vatajakasa, Vataja atisara, Vataja visarpa, Vataja hridroga -28

183. Ruksha madyapana:Mutrakricchra -1

184. Ruksha pana:Gulma, Vataja Gulma, Karshya--3

185. Sailamamsa:Raktadusti--1

186. Saka:Kaphaja prameha, Doshaja arsa --2

187. Saktu:Raktadusti --1

188. Saluka:Hikkaswasa, Doshaja Arsa -2

189. Samala Ahara: Unmada, Apasmara $-2$

190. Samasnata sarva rasa:Sannipatika chardi --1

191. Sampurana:Kaphaja unmada --1

192. Samsrusta anna:Nijaswayathu --1

193. Sankeerna asana:Krimija siroroga, Doshaja arsa--2

194. Sanklista Ahara:Antarvidradhi, Beejopaghataja klaibya --2

195. Sarkara/Trina/Kasta/Asthi/Kantaka with Anna:Chidrodara --1

196. Sarshapa taila siddha rohinisaka, kanakapota:Raktapitta --1

197. Sarshapa:Raktapitta --1

198. Seeta Ahara while jeernajeerne samsnata:Urustambha --1

199. Seeta:Vataja Jwara, Kaphaja Jwara, Kaphaja Gulma, VatajaPrameha, Sannipataja Atisara, Beejopaghataja Klaibya, Ananatavata, Antarvidradhi, Vataja unmade, Vataja arsa, Kaphaja arsa, Kaphaja grahani, Vataja Grahani, Vataja kasa, Kaphaja atisara, Grandhi visarpa -- 16

200. Sigru: Raktapitta --1

201. Seetaanna:Doshaja arsa --1

202. Siramamsa: Doshaja arsa--1

203. Sleshmala Ahara:Hikkaswasa--1

204. Sneha pana-atyambupana:Jalodara $-1$

205. Snehapana:Urustambha, Doshaja Arsa --2 
206. Snigdha Ahara while jeernajeerne samsnata:Urustambha

207. Snigdha:Kaphaja Gulma, Kusta, Sannipataja Atisara, Vatarakta, Raktapradara, Sthoulya, Tandra, Kaphaja udara, Kaphaja arsa, Kaphaja Grahani, Kaphaja kasa, Kaphaja atisara, Kaphaja chardi, Grandhi visarpa, Kaphaja visarpa, Kaphaja hridroga-- 16

208. Souktika with unboiled milk: Raktapitta--1

209. Souktika: Raktapitta --1

210. Souveeraka:Raktapitta, Visarpa, Rakta Dusti, Vatarakta--4

211. Sringataka: Doshaja arsa --1

212. Sthira: Grandhi visarpa--1

213. Sugandhaka: Kaphaja Prameha--1

214. Sukta:Raktapradara, Vatarakta, Visarpa --3

215. Sumukha: Raktapitta --1

216. Sura: Raktapitta, Visarpa, Raktapradara, Raktadusti, Vatarakta $--5$

217. Surasa: Raktapitta --1

218. Surasava: Raktapitta --1

219. Sushka Ahara:Anatarvidradhi, Urustambha, Trishna, Vataja hridroga--4

220. Sushka mamsa:Vatarakta, Doshaja arsa --2

221. Sushkasaka:Doshaja Arsa, Raktapitta --2

222. Taking Kakamachi while indigestion: Kusta --1

223. Taking lakucha while indigestion: Kusta --1

224. Taking madhu while indigestion:Kusta --1

225. Taking matsya while indigestion: Kusta --1

226. Taking mulaka while indigestion:Kusta --1

227. Taking phanita while indigestion:Kusta --1

228. Takra with Kola/kuluttha/masha/atasi/kusumbha sneha:Kusta --1

229. Takra:Vatarakta --1
230. Takrapinda:Doshaja arsa--1

231. Taruna dhanya:Kaphaja prameha--1

232. Taruta: Doshaja arsa --1

233. Teekshna : Raktapitta, Pittaja gulma, Nija swayathu, Hridroga, Raktadusti,Pittaja udara, Pittaja arsa, Pittaja atisara --8

234. Teekshna madya:Vataja arsa, Vataja atisara --2

235. Teekshnoushadha:Mutrakricchra, Pittaja arsa, Vataja chardi --3

236. Tiktarasa:Vataja prameha, Vatarakta, Udavarta, Sukradusti, Vataja arsa, Vataja grahani --6

237. Tila taila:Pandu, Hikkaswasa --2

238. Tila:Kaphaja Prameha, Kusta, Doshaja Arsa, Visarpa, Krimija Siroroga --5

239. Tilaksheera:Kusta --1

240. Tushodaka: Raktapitta --1

241. Udaka:Prameha, Kaphaja prameha, Visarpa, Urustambha, Nijaswayathu, Raktapradara, Stanyadosha, Dhwajabhangaja klaibya --8

242. Uddalaka:Raktapitta, Kaphaja Prameha, Kusta --3

243. Udwasit-processed With Yavaka, Uddalaka, Kora Dusha, Nishpava, Masha, Kuluttha-Raktapitta --1

244. Upaklinna anna:Vatarakta, Raktadusti --2

245. Upavasa:Anantavata, Vataja unmada, Vataja chardi, Vataja hridroga--4

246. Ushna Ahara while jeernajeerne samsnata:Urustambha --1

247. Ushna : Pittaja Jwara, Pittaja Gulma, Pittaja Prameha, Raktapitta, Nija Swayathu, Udara, Pandu, Sannipataja Atisara, Visrapa, Trishna, Vatarakta, Sukra Dusti, Antarvidradhi, Raktadusti, Pittaja unmada, Pittaja udara, Pittaja Arsa, Pittaja kasa, Pittaja atisara, Pittaja chardi, Vataja visarpa, Pittaja Visarpa, Pittaja hridroga -- 23

248. Ushna raga:Visarpa --1

249. Ushnabhitapta paya pana:Raktapitta $--1$ 
250. Ushnabhitapto va atimatram ativelam paya pana: Raktapitta --1

251. Vaishamyayuktena Aharopayoga: Unmada, Apasmara --2

252. Varaha mamsa- processed with Yavaka, Uddalaka, Kora Dusha, Nishpava, Masha, KulutthaRaktapitta -- 1

253. Varaha mamsa: Doshaja arsa --1

254. Vasa: Doshaja arsa--1

255. Vidagdahara sevana without doing vamana of vidahikara Ahara:Kusta

256. Vidahi:Raktapitta, Pittaja Gulma, Doshaja Arsa, Hikkaswasa, Visarpa, Raktapradara, Antarvidradhi, Udara, Pittaja unmada, Pittaja udara, Pittaja Arsa, Pittaja grahani, Pittaja kasa, Pittaja chardi, Kaphaja chardi, Pittaja visarpa -- 16

257. Vikrita Ahara:Unmada, Apasmara -2

258. Vilepi:Kaphaja prameha --1

Switra, Unmada, Swayathu, Udara, DoshajaArsas, Pandu, Sannipataja Atisara, Visarpa, Vatarakta, Beejopaghataja Klaibya, Dhwajabhanja Klaibya, Stanya Dosha, Antarvidradhi, Raktadusti -15

260. Virudha Dhanya:Arsa --1

261. Virudha nava samidhanya: Kaphaja Prameha --1

262. Virudha nava suka dhanya: Kaphaja Prameha --1
263. Virudha Samidhanya:Nija Swayathu $--1$

264. Vishamasana:Pittaja Jwara, Dwandwa Sannipataja Jwara, Rajayakshma, Agnidusti, Hikkaswasa, Stanya Dusti, Kshayaja Kasa, Sannipataja Atisara --8

265. Vishopahatasya vishasya upayoga: Dwandwasannipataja jwara --1

266. Vistambhi:Hikkaswasa --1

267. Vyapanna madya:Antarvidradhi, Urustambha, Visarpa --3

268. Vyapanna salilapana:Doshaja arsa $-1$

269. Yavaka: Kaphaja Prameha, Raktapitta, Kusta.---3

Total 269 Aharaja nidana were mentioned for 91 disease conditions.

Top 10 Aharaja nidana competent to produce highest number of diseases are:

- Ruksha guna Ahara -28

- Katu rasa -23

- Ushna guna Ahara -23

- Lavana rasa -21

- Guru ahara -20

- Ajeernasana -18

- Vidahi -16

- Snigdha -16

- Seeta -16

- Viruddhahara -15

\section{Top 10 Aharaja nidana}

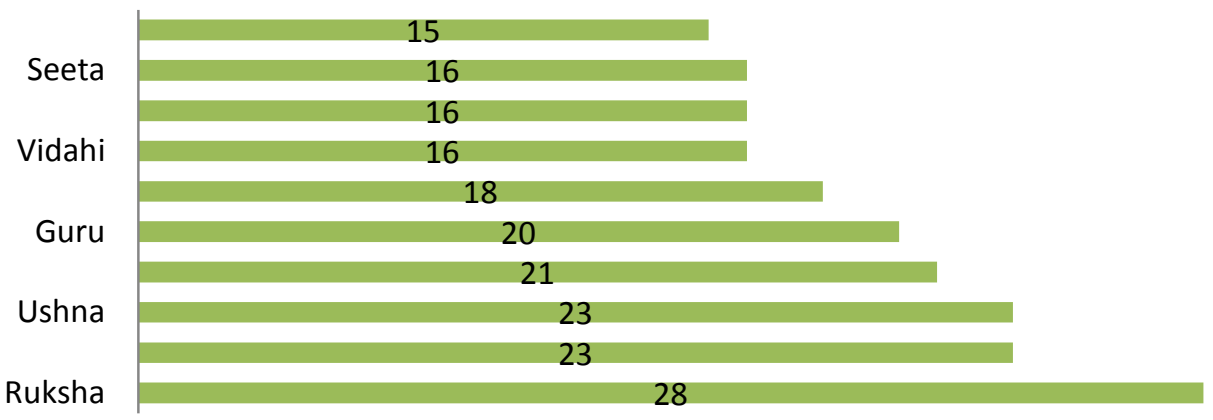




\section{Discussion :}

An overall analysis on the various contributing factors of the diseases, had established that $97 \%$ of the diseases are due to Aharaja nidana. As it was quoted by Charaka "Ahara sambhavam vastu rogascha ahara sambhava(4)". Ahara is a key factor for both health and disease.It was said that it is the need of the hour to concentrate on Nidana Parivarjana chikitsa.This line holds good in satisfying that $97 \%$ of Nidana is from Aharaja group.

Aharaja group not only involves Ahita Ahara but also includes correction in Dietary habits (Vishamasana, Samasana, Viruddhasana etc., ) also.

Among 94 diseases mentioned in Charaka samhita except 3 diseases all other 91 diseases have established $97 \%$ of involvement of Aharaja nidana.

Viharaja nidana contributes for 79 (84\%), Manasika nidana 77 (88\%), Others $42(44.6 \%)$ disease conditions.

Listing of Top 10 Aharaja nidana reveals that maximum number of Pitta disorders Raktapradara(100\%), Pittaja Visarpa(100\%), Pittaja Chardi(100\%), Pittaja Grahani(100\%), Pittaja Unmada(100\%), Rakta pitta (98.33\%)are caused due to predominancy of Aharaja nidana. This shows that more number of Pitta disorders occurs due to intake of Pitta vardhaka ahara.

Listing of Top 10 Viharaja nidana shows the relevance of involvement of Viharaja nidana in involvement of Kshataja kasa(100\%), Kshataksheena(100\%). Maximum number of Siroroga are due to Viharaja nidana i.e Ardhavabhedka (67\%), Siroroga(56\%), Suryavarta(50\%), Vataja siroroga (50\%), Kaphaja siroroga (40\%) and Pratisyaya $72 \%$.

The psychological status has influence on Impotency which was supported by involvement of Manasika nidana in Sukrakshayaja klaibya(62\%),
Beejopaghataja Klaibya(24\%), Skin disorders (Switra-57\%), Psychological disorders (Apasmara-48\%, Unmada-44\%) and heart diseases (Hridroga-23\%)

It was observed that Ruksha guna Ahara is capable to produce highest number of diseases i.e., 28 followed by Katurasa (23) which is supportive to the highest number of Vataja nanatmaja vyadhis (80).

Ushna guna Ahara, Lavana rasa, Ajeernasana, Vidahi Ahara capable to produce 23, 21, 18, 16 number of diseases respectively supportive to the number of Pittaja nanatmaja vyadhis(40).

Similarly Snigdha, Seeta, Viruddhahara, Dadhi produces 16, 16, 15, 14 number of disorders respectively.

\section{Conclusion:}

The above study clearly stated among all the various groups of factors involved in disease manifestation the major portion is contributed by Aharaja nidana followed by Viharaja, Manasika and other factors. Out of all Nidana, Ahara has to be taken care of owed to more number of factors getting to be vitiated.Catalogue provides a directive like dictionary to the entire causative factors (Aharaja nidana) mentioned in Charaka Samhita and diseases caused by them. This serves as a guidelines for the probability of getting maximum number of diseases by a single factor. The individual role played by diet and dietary habits in each disease can be understood.

Actually this is a small study pertaining to Charaka Samhita focussing only the involvement of Aharaja nidana in disease manifestation. It needs further deep analysis relating to each and every factor in disease manifestation mentioned in different classical literature and possible correlation of the said Aharaja nidana in the modern day dietetics. 


\section{References:}

1. Yadavji Trikamji, Charaka Samhita by Agnivesa, Revised by Charaka and Dridhabala, $4^{\text {th }}$ edition, Newdelhi, Munshiram manoharlal publishers pvt limited, 1981, 74p.

2. Yadavji Trikamji, Charaka Samhita by Agnivesa, Revised by Charaka and Dridhabala, $4^{\text {th }}$ edition, Newdelhi, Munshiram manoharlal publishers pvt limited, 1981, 76p.
3. SrikantaMurthy KR, Susrutha Samhita, volume-3, $4^{\text {th }}$ edition, Varanasi, Chaukambha orientalia, 2010,7p

4. Yadavji Trikamji, Charaka Samhita by Agnivesa, Revised by Charaka and Dridhabala, $4^{\text {th }}$ edition, Newdelhi, Munshiram manoharlal publishers pvt limited, 1981, 181p. 